\title{
Divulgación de información sobre el capital intelectual de empresas nacionales que cotizan en la Bolsa Mexicana de Valores
}

Ruth Leticia Hidalgo

\section{Gallardo}

Profesora, Instituto de Ciencias Económico Administrativas, Universidad Autónoma del Estado de Hidalgo ruth.757@hotmail.com

\section{Emma García Meca}

Profesora titular, Departamento de Economía Financiera y Contabilidad, Facultad de Economía y Empresa, Universidad de Murcia emmagar@um.es

\section{Resumen}

Con la nueva visión económica se reconoce al capital intelectual como una herramienta de creación de valor para las empresas que buscan una mayor competitividad en el mercado. En este trabajo se analiza la divulgación que en materia de intangibles llevan a cabo las empresas mexicanas, así como los factores que motivan tal divulgación; para ello, se analizaron los reportes de los anuales de cien empresas que cotizan en la Bolsa Mexicana de Valores. Los resultados reflejaron que para las empresas mexicanas es más importante la divulgación de información del capital estructural, seguido del capital relacional y posteriormente del capital humano; el tamaño de la empresa es el factor clave de la divulgación. De igual forma, se encontró que las empresas de los sectores: industrial, de alimentos y bebidas, y construcción e inmobiliaria son proactivos en el momento de divulgar información sobre intangibles.

Palabras clave: capital intelectual, revelación de información de intangibles, empresas mexicanas que cotizan en la Bolsa Mexicana de Valores. 


\title{
Disclosure of information on intellectual capital in Mexican firms listed on the Mexican Stock Exchange.
}

\begin{abstract}
Intellectual capital is at present time a value-creating tool for companies seeking better competitiveness. This paper analyzes intangibles disclosure in Mexican companies listed on the stock market, as well as the factors influencing such disclosure. For the accomplishment of this study annual reports from one hundred Mexican companies listed on the Mexican Stock Exchange were analyzed. Findings showed that it is more important for Mexican companies the disclosure of structural capital, followed by relational capital, and finally human capital, with company size being the key factor in such information. Likewise, it was found that enterprises in industry, food and beverage, and building and real estate sectors are proactive when disclosing information on intangibles.
\end{abstract}

Keywords: intellectual capital, disclosure intangible, annual report, mexican companies stock market.

\section{Introducción}

De acuerdo con los conceptos de competitividad internacional de las empresas y su posicionamiento en el mercado, es necesario considerar la importancia de la información sobre los activos intangibles o capital intelectual como generadores de valor de la empresa. Esta información se ha convertido en prioritaria para los inversionistas, gerentes, analistas financieros, analistas de riesgo y, en general, toda la comunidad interesada en el funcionamiento empresarial.

En la Norma Internacional de Contabilidad (NIC) 38, el activo intangible se define como un bien "identificable, de carácter no monetario y sin apariencia física, que se utiliza en la producción o suministro de bienes y servicios, arrendarlo a terceros o para funciones relacionadas con la entidad". Esta definición enmarca requisitos que restringen el reconocimiento del activo intangible dentro de la información financiera, lo que implica que sólo se reflejen los intangibles adquiridos (Cañibano et al., 2008). Por esta razón, la tendencia de los organismos reguladores internacionales, como el Financial Accounting Standard Board (FASB) o el International Accounting Standard Board (IASB) consiste en recomendar la inclusión de información adicional sobre activos intangibles fuera de los estados financieros, evitando así la incorporación de criterios contables que puedan poner en riesgo la 
calidad y fiabilidad de la información financiera. Las empresas pueden completar la información sobre sus activos intangibles mediante su revelación voluntaria.

Existe evidencia empírica que países europeos y Estados Unidos de América (EUA) han llevado a cabo estudios que analizan cómo las empresas revelan datos sobre su capital intelectual. Contrariamente, en México no hay estudios sobre la forma en que las empresas manejan estos activos. Es por ello que en este trabajo nos proponemos investigar hasta qué punto las empresas mexicanas llevan a cabo la divulgación voluntaria de datos sobre su capital intelectual dentro de sus reportes anuales y qué factor o factores influyen en la misma. Para lograr este objetivo, estudiamos la influencia de los activos intangibles considerando aspectos como: tamaño de la empresa, cotización en mercados extranjeros, sector al que pertenecen, endeudamiento, rentabilidad y existencia de pérdidas económicas. Esto se desarrollará mediante el análisis de la información que las empresas mexicanas otorgan al cotizar en la Bolsa Mexicana de Valores (BMV). Debido a la ausencia de información de los activos intangibles en este país, analizaremos si las empresas mexicanas identifican al capital intelectual como una herramienta útil para crear ventaja competitiva en su desarrollo y actividad comercial.

Para explicar lo anterior, en la siguiente sección, se hace referencia a determinados estudios que en materia de divulgación del capital intelectual se han llevado a cabo; más adelante se establece la metodología de la investigación: el objetivo, la muestra, las hipótesis y la metodología utilizada para el estudio empírico; posteriormente, se presentan los resultados obtenidos; por último, se exponen las conclusiones del trabajo.

\section{Estudios sobre divulgación de información del capital intelectual}

El capital intelectual es una característica innata de la empresa, generada en el transcurso del tiempo mediante experiencias, estrategias, toma de decisiones, gestiones y cultura, cualidades que deben sostenerse, mantenerse y acrecentarse. Tales acciones se traducen en "creación de valor" y "ventaja competitiva", las cuales representan el recurso estratégico para que las empresas compitan en la nueva economía.

Comúnmente, se emplean las siguientes categorías propuestas por Edvisson y Malone (1997), Euforum (1988), Cañibano et al. $(2002,2008)$ para clasificar los elementos que configuran el capital intelectual. 
1. Capital humano. Son las capacidades individuales, conocimientos, destreza y experiencia de los empleados y directivos de la empresa. Incluye aspectos tales como la formación de sus empleados, ingresos por empleado o experiencia y calidad del equipo directivo, entre otros.

2. Capital estructural. Se define como el conjunto de conocimientos que permanece en la empresa al final del día. Incluye las patentes, ideas, estructuras de funcionamiento y la organización administrativa e informática de la empresa. Además, incluye aspectos sobre la capacidad instalada, eficiencia productiva e incluso la gestión interna del negocio, cultura corporativa o la estrategia de comunicación en la compañía. Dentro de este bloque se observan los procesos de trabajo, las técnicas y programas para empleados que aumentan y fortalecen la eficiencia de producción o la prestación de servicios, así como aspectos relativos a las herramientas que aceleran la corriente de conocimientos a través de la organización.

3. Capital relacional. Se define como el conjunto de recursos ligados a las relaciones externas de la empresa, es decir, con sus clientes, proveedores, accionistas o socios de $\mathrm{I}+\mathrm{D}$, de los que se obtienen aspectos relativos a la dependencia de clientes clave, ventas por cliente o descripciones del compromiso y relaciones con clientes o proveedores.

Esta clasificación hace posible la identificación y medición del capital intelectual para analizar a la empresa tanto interna como externamente. Navas y Ortiz de Urbina (2002) afirman que no ha existido un criterio unificado para la elaboración de la clasificación de los activos intangibles debido a que no existe un análisis profundo de los mismos; también estos autores señalan la necesidad de catalogar a dichos activos en distintas clases, lo que permitiría caracterizarlos y analizarlos según su contribución a la competitividad; es decir que sean aspectos relevantes al momento de soportar una ventaja competitiva.

Los indicadores que utilizamos para medir el capital estructural, relacional y humano en las empresas mexicanas se basó en los modelos: Edvissson y Malone (1997), denominado "Navegador de Skandia"; Sbeiby (1997), designado como "Intangible Assets Monitor"; y Cañibano et al. (2002) nombrado "Méritum". Éstos se caracterizan por su enfoque, flexibilidad y múltiples opciones que permiten apreciar cada aspecto de la entidad. 
Considerando los estudios previos que han investigado el papel del capital intelectual, encontramos que una de las líneas de investigación más prolíferas consiste en determinar qué factor o factores influyen para que las empresas divulguen intangibles. Esto es estudiado por Teece (2000), Lev (2000), Bueno et al. (2004), Gray et al. (2004), Cañibano (2005) y García-Meca (2005). Conociendo la problemática de que los activos intangibles no se encuentran reflejados en los estados financieros por no cumplir con los requisitos de reconocimiento y medida de acuerdo con la normativa contable, las empresas determinan informarlo de manera voluntaria.

La mayoría de los investigadores que han estudiado la revelación voluntaria de datos de los activos intangibles se han basado en las empresas que cotizan en la bolsa, porque éstas se publican en las páginas web, lo cual es una vía de fácil acceso para la obtención de los reportes anuales, además de que la información que se audita brinda la confianza de su veracidad.

Los resultados de los estudios en materia de divulgación de capital intelectual se han llevado a cabo por autores como García-Arrieta (2001), García-Meca (2005) y Abad et al. (2007). Estos estudios, desarrollados en España, mostraron una escasa información voluntaria porque solamente consideraron dentro de los reportes anuales aquella información que era obligatoria. Asimismo, estos trabajos empleaban índices de divulgación para medir la cantidad de información revelada de forma voluntaria, donde el factor tamaño era decisivo en la divulgación de los activos intangibles.

Los estudios elaborados por Laínes et al. (1999), Villacorta (2005) y Monclús et al. (2007) dirigen su planteamiento a la divulgación del recurso humano considerando a las empresas establecidas en países de la Unión Europea (UE). Estos análisis determinan que las empresas refieren el recurso humano en las cuentas anuales. De igual manera, las empresas registran el capital humano en diferentes ubicaciones y con diferente nomenclatura, apareciendo con una profundidad desigual en los informes anuales. En resumen, los indicadores y los apartados para medir el capital humano deben normalizarse para cada sector, lo que permitirá la existencia de una comparación interempresarial. En relación con los ítems analizados, éstos revelan que las categorías más divulgadas son: perfil del empleado, seguido de la formación permanente y la renovación educativa, por lo que estos autores argumentan que las empresas estudiadas en muchos casos presentan informes voluntarios dedicados exclusivamente a notificar políticas de recursos humanos; en consecuencia, señalan que el principal factor explicativo sobre la cantidad de información sumi- 
nistrada es el tamaño de la empresa, pues entre más grande es ésta mayor cantidad de información sobre capital humano proporciona.

De igual forma, es importante considerar el estudio realizado por Rodríguez (2004), quien examinó los factores que influían en la revelación voluntaria de información con respecto a la ventaja competitiva empresarial; tales factores son analizados mediante los informes anuales de empresas que cotizan en el mercado

Continuo español (Sistema de Interconexión Bursátil Español, SIBE) en 2000. Asimismo, Rodríguez analizó las fuentes de ventaja competitiva desde las teorías de recursos y capacidades; encontró que la cantidad de información revelada sobre fuentes de ventaja competitiva no es demasiado alta, pues reflejaba un total de $44.9 \%$ con un máximo de $38 \%$ y un mínimo de $18 \%$, frente a otros estudios que indicaban que se encontraban entre el $60 \%$ con mínimos del $40 \%$ y máximos del $80 \%$. Es posible que esto se deba al número de ítems considerados, o a que la empresa no revela este tipo de información que es clave y que puede ser utilizada por sus competidores.

Igualmente, Rodríguez determinó que las variables "tamaño de la empresa” y "sector" son las que mejor explican la extensión de la información que revela una empresa y son reflejados en todos los índices analizados; "rentabilidad" y "acciones en manos de los seis principales accionistas" son variables que explican la revelación de información sobre capital humano; por otro lado, "cuota de mercado" explica la revelación de información sobre capital relacional.

Con la finalidad de obtener opiniones empresariales del modelo para medir el capital intelectual, Devece et al. (2007), con la metodología Delphi, llevaron a cabo su estudio mediante encuestas a empresarios de diferentes sectores pertenecientes al Club de Gestión del Conocimiento e Innovación de la Comunidad Valenciana, donde el conocimiento es considerado como recurso clave. Concluyen que actualmente no se cuenta con un modelo aceptado por ninguna estancia, ya sea la comunidad científica o empresarial, para la medición del capital intelectual (humano, organizativo y relacional) porque la mayoría de dichos modelos no están concluidos y la medición no es clara y precisa.

Dadas las circunstancias anteriores de divulgación de activos intangibles por parte de las empresas, Castilla y Gallardo (2007), al hacer una revisión exhaustiva a la literatura, buscaban conocer los siguientes aspectos: tipo de información revelada, medio utilizado e informes presentados. En su documento "Estado de capital 
intelectual" señalaron que para la divulgación del capital intelectual es necesaria la estandarización de la información. Para el caso español propusieron el modelo 3R, que estaba integrado por el informe de capital intelectual, la cuenta de flujos de capital intelectual y la memoria de capital intelectual. También señalaron que para tal efecto contaban con un número considerable de directrices que las empresas podrían utilizar. Conforme transcurra el tiempo, se vera qué directrices resultan viables y se promoverá un proyecto de homogeneización.

\section{Metodología de la investigación}

\section{Objetivos y formulación de hipótesis}

La realización del estudio empírico tiene como objetivo conocer si las empresas mexicanas que cotizan en la Bolsa Mexicana de Valores (BMv) divulgan la información del capital intelectual dentro de su reporte anual como una estrategia para incursionar en el mercado. Específicamente nos interesa saber:

- En qué medida las empresas revelan, dentro de sus estados financieros, el capital intelectual y si la carencia de información se debe a la falta de normatividad existente en México.

- Analizar si la divulgación del capital intelectual está en función del tamaño de la empresa, tipo de sector al que pertenece, endeudamiento, rentabilidad financiera o cotización en otras bolsas.

Mediante la verificación de los informes anuales de las empresas se podrá conocer la cantidad de información que están dispuestas a divulgar y los factores explicativos de esta revelación. A este respecto se han elaborado las siguientes hipótesis.

H1: Las empresas mexicanas que cotizan en el mercado de valores divulgan información de capital intelectual en función a su tamaño.

De acuerdo con la teoría de agencia (Jensen y Mecking, 1976; Myers, 1977) y la teoría de las señales (Ross, 1979) existe una relación positiva entre el tamaño de la empresa y la extensión de la información divulgada. Los trabajos de Monclús et al. (2007) y Rodríguez (2004) muestran que el principal factor explicativo sobre información revelada es el tamaño de la empresa. Esto se debe a que existe una 
mayor necesidad de obtener fondos del exterior, procesamiento e información de las grandes empresas, seguimiento de inversionistas y analistas sofisticados, además de la consecuencia de una mayor presión por parte de accionistas y analistas sobre información detallada de los conductores de valor de estas empresas. Por las razones anteriores, consideramos que las empresas de mayor tamaño divulgarán más información sobre los activos intangibles.

H2: Las empresas mexicanas que cotizan en el mercado de valores divulgan información de capital intelectual en función al sector productivo al que pertenecen.

La teoría de las señales indica que la divulgación de la información de los activos intangibles por la empresa está condicionada por el sector al que pertenecen. Los trabajos de Adrem (1999) y Meek et al. (1995) encuentran una influencia significativa del sector de las empresas que divulgan los activos de forma voluntaria. En este sentido, la variable sector la consideramos como un factor determinante de la cantidad y especificidad en información divulgada sobre capital intelectual.

H3: Las empresas mexicanas que cotizan en el mercado de valores divulgan información de capital intelectual en función a su cotización en mercados extranjeros.

Los requisitos de información para cotizar en mercados de capitales extranjeros hacen de éste un factor relacionado con la estrategia de divulgación voluntaria de la empresa (Cooke, 1989). Las empresas que necesitan de una mayor financiación requieren mantener una estrategia de comunicación de mayor calidad y transparencia con el objeto de mejorar su imagen en el nivel internacional, bajar sus costos de capital, reducir la incertidumbre, así como el aumento de la contratación de sus títulos.

H4: Las empresas mexicanas que cotizan en el mercado de valores divulgan información de capital intelectual en función a la existencia o no de pérdidas.

Se estima que las empresas que presentan pérdidas en sus informes financieros divulguen información de los activos intangibles y, de esta manera, darán seguridad a sus inversionistas; en este planteamiento estaríamos considerando la teoría de las señales.

H5: Las empresas mexicanas que cotizan en el mercado de valores divulgan información de capital intelectual en función a la rentabilidad financiera. 
La teoría de los recursos y capacidades contempla que los recursos organizativos podrían presentar una ventaja competitiva sostenida por la empresa convirtiéndose, de este modo, en recurso estratégico. Asimismo, desde la perspectiva de la teoría de las señales, las empresas con alta rentabilidad pueden dar a conocer su buena situación dentro del mercado.

H6: Las empresas mexicanas que cotizan en el mercado de valores divulgan información de capital intelectual en función de su nivel de endeudamiento.

La teoría de agencia considera que la divulgación de los activos intangibles es una herramienta para reducir los costos de agencia, pues contribuye en la reducción de asimetrías informativas entre los participantes. De esta manera disminuye el riesgo promedio y aumentan las garantías como deudores, provocando que los acreedores aumenten los niveles de crédito y disminuyan el tipo de interés. La evidencia empírica de Harris y Raviv (1991), Stulz (1990), Frank y Goyal (2003) vislumbra la existencia de una menor presencia de deuda en las empresas que divulgan más sus activos intangibles.

\section{Metodología}

La muestra de este estudio está formada por empresas no financieras mexicanas que cotizaron en la Bolsa Mexicana de Valores durante 2005. En el cuadro 1 se detalla la distribución de la información considerando una muestra final de cien empresas.

\section{Cuadro 1}

Empresas nacionales que cotizan en la Bolsa Mexicana de Valores

\begin{tabular}{lc}
\hline \multicolumn{1}{c}{ Concepto } & Total de empresas \\
\hline Total de las empresas emisoras & 137 \\
Empresas excluidas por emisoras extranjeras & 3 \\
Emisoras que no cuentan con información & 16 \\
correspondiente a 2005 & 18 \\
Emisoras financieras y de seguros & $\mathbf{1 0 0}$ \\
\hline Total empresas incluidas en la muestra & \\
\hline
\end{tabular}

Fuente: Elaboración propia según información de la Bolsa Mexicana de Valores 
En el cuadro 1 se presentan 137 empresas que cotizaron en 2005, de las cuales se excluyó un $0.02 \%$ de empresas de capital extranjero que cotizan en México; de igual forma, se prescindieron de aquellas empresas que en ese mismo año no contaban con información financiera, las cuales representan 12\%; asimismo, se descartó $13 \%$ correspondiente a empresas que poseían capital ajeno, como son las instituciones financieras y de seguros. Después de aplicar los filtros anteriores, se obtuvo un total de cien empresas por analizar que representan $73 \%$ de las empresas emisoras.

Una vez que se seleccionaron las empresas, se buscaron los informes anuales, los cuales están estructurados de acuerdo con la información general, la historia de la compañía, la información financiera, administración y anexos. Con esto se determinaron la variable dependiente y la variable dicotómica, considerando el valor de 1 si las empresas divulgan capital intelectual y el valor 0, en caso contrario. Esto se realizó mediante la lectura y análisis de los reportes anuales correspondientes a 2005, determinando cada uno de los índices correspondientes al capital estructural, capital humano y capital relacional, con un total de 58 ítems (véase Anexo 1). La clasificación de los activos intangibles en las tres categorías anteriores se ha realizado siguiendo la literatura empírica previa (Edvinsson y Malone, 1997; Euroforum, 1998 y Cañibano et al., 2002).

Para la selección de los distintos ítems sobre capital intelectual se consideraron los indicadores de los modelos ya mencionados de Skandia (1997), el Monitor de Sveiby (1997) y Meritum (2002), así como los trabajos elaborados por GarcíaMeca et al. (2005) en España y Bukh et al. (2001) en Dinamarca; asimismo, se incorporaron aquellos ítems que, de forma muy particular, las empresas mexicanas divulgaron. La metodología utilizada por estos autores permite obtener resultados más objetivos; de igual manera, permite trabajar con una muestra más amplia que refleje los diferentes comportamientos de las variables por analizar; además, es la más usual para este tipo de estudios, los cuales suelen publicarse en revistas importantes del área. Otras metodologías son las encuestas o el estudio del caso; no obstante, éstas presentan algunos problemas entre los que se encuentran: mayor riesgo de sesgo muestral, problemas de respuesta; además, sus resultados suelen ser subjetivos, porque pueden estar basados en sentimientos personales como la intuición y no en la observación o razonamiento; otra limitante es que el estudio de caso se enfoca hacia un solo individuo u objeto. Sin embargo, no se descarta este tipo de métodos para aplicarlos en futuras investigaciones de forma complementaria a nuestra metodología. 
Por otra parte, y una vez trabajadas las hipótesis, se determinaron a determinar las variables independientes y, al mismo tiempo, se fijaron los códigos por utilizar, cómo se muestra en el cuadro 2.

Cuadro 2

Variables independientes

\begin{tabular}{ll}
\hline Variables & Códigos \\
\hline Sector productivo & Sector \\
Cotiza mercado extranjero & CotMExt \\
Cifra de negocio & LnCNeg \\
Activo & Act \\
Variable pérdidas & Var.Pérdidas \\
Rentabilidad financiera & R.Fin \\
Endeudamiento & Ende \\
Número de empleados & NE \\
\hline
\end{tabular}

Fuente: Elaboración propia

Las variables independientes tuvieron una importancia relevante para el desarrollo de este estudio, por lo que su descripción es elemental para el análisis, el cual se detalla a continuación.

- Como variables de tamaño se consideraron los siguientes aspectos:

Cifra de negocio ( $\mathrm{LnCNeg}$ )

Activo (Act)

Número de empleados (NE)

- Sector productivo (Sector). Los sectores considerados para las empresas se elaboraron de acuerdo con la actividad que desarrollaban, tomando en cuenta los sectores alimentos y bebidas, construcción e inmobiliaria, industrial, comunicación, textil, calzado y complementos y otros. Esta variable dicotómica considera el valor de 1 , si la empresa pertenece al sector identificado y un valor de 0 si no pertenece a ese sector.

- Cotización en mercado extranjero (CotMExt). Variable dicotómica que mostrará el valor de 1, si la empresa cotiza en otro mercado bursátil internacional y 0 si no cotiza en otro mercado.

- Variable pérdidas (Var.Pérdida). Se refiere al beneficio neto de la entidad; es una variable dicotómica a la cual corresponde el valor 1 , si tiene pérdidas y valor 0 , si tienen utilidad. 
- Rentabilidad financiera (R.Fin). Variable que se determinó con el cociente resultante de dividir el beneficio neto entre los fondos propios.

- Endeudamiento (Ende). Al igual que en el caso anterior, la variable fue determinada como el cociente entre pasivo y activo.

\section{Presentación de resultados}

\section{Análisis descriptivo del capital intelectual}

Después de haber elegido las empresas y las variables mediante las que se determinarían los fenómenos cuya inferencia se desea contrastar, procedemos a detallar las técnicas de análisis mediante el programa de SPSs, donde se muestre la información estadística correspondiente a cada rubro de acuerdo con los objetivos delimitados.

En primer lugar, se llevó a cabo un análisis descriptivo del capital intelectual, que muestra las estadísticas de resumen univariado, así como valores tipificados por cada índice de divulgación, lo cual nos permitió conocer adecuadamente las variables sometidas a prueba, determinando su naturaleza métrica; con ello pretendimos obtener información que mostrara qué ítems son los más divulgados por las empresas mexicanas y qué clasificación es la más representativa, lo cual se muestra en el siguiente cuadro:

\section{Cuadro 3}

Estadísticas descriptivas del capital intelectual por grupos

\begin{tabular}{lccccc}
\hline & $\mathrm{N}$ & Mínimo & Máximo & Media & Desv. Típ. \\
\hline Capital estructural & 100 & 8 & 22 & 16.51 & 2.765 \\
Capital relacional & 100 & 3 & 16 & 9.76 & 2.913 \\
Capital humano & 100 & 1 & 14 & 6.73 & 2.891 \\
\hline
\end{tabular}

Fuente: Elaboración propia siguiendo los resultados de sPss

Este análisis estadístico de la información divulgada referente al capital intelectual, que se refleja en el cuadro, indica que el capital estructural posee el mayor porcentaje de divulgación (16.51), seguido por el capital relacional (9.76) y finalmente el capital humano (6.73). Con respecto a estos resultados, a continuación se reflejan los datos estadísticos descriptivos de cada uno de ellos para así determinar los ítems que conforman la clasificación del capital intelectual. 


\section{Cuadro 4 \\ Revelación de información sobre capital estructural}

\begin{tabular}{lc}
\hline & Frecuencia \\
\hline Riesgos de la empresa & 1.00 \\
Capacidad instalación de punta & .97 \\
Gobierno corporativo & .95 \\
Calidad de sus productos & .95 \\
Cuota de mercado & .94 \\
Estructura control interno & .91 \\
Inf. imagen líder marcas & .91 \\
Estructura organizativa & .90 \\
Estructura accionarial & .88 \\
Gestión del modelo negocio & .88 \\
Eficiencia productiva & .85 \\
Diversidad nuevo productos tecnológicos & .78 \\
Políticas / normas medioambientales & .77 \\
Ventaja competitiva & .68 \\
Utilización energía y otros inputs & .63 \\
Responsabilidad social corporativa & .61 \\
Reconocimiento activos intangibles & .56 \\
Sistemas e inversiones de IT & .54 \\
Certificados y reconocimientos de calidad & .48 \\
Políticas objetivos estratégicos I+D+I & .43 \\
Información visión del negocio & .38 \\
Sistemas integrales de información & .32 \\
Servicios sociales & .19 \\
\hline
\end{tabular}

Fuente: Elaboración propia siguiendo los resultados de sPss

Para las empresas mexicanas es importante dar a conocer en primer lugar el aspecto interno que es el capital estructural. El cuadro 4 explica estadísticamente que los ítems que ocupan él más alto porcentaje de divulgación van de un 95\% a 100\%, los cuales se refieren a riesgos de la entidad, capacidad de instalación de punta, gobierno corporativo y calidad de productos.

Es elemental mencionar que entre los índices más significativos se encuentran el de gobierno corporativo, que es un aspecto que en los últimos años ha tenido gran importancia y que es considerado también por las empresas mexicanas. Los ítems menos reconocidos se encuentran por debajo del 50\% dentro de los cuales se localizan los certificados de reconocimientos de calidad; políticas, objetivos, estra- 
tegias de investigación, desarrollo e innovación; información sobre la visión del negocio; sistemas integrales de información y servicios sociales.

\section{Cuadro 5 \\ Revelación de información sobre capital relacional}

\begin{tabular}{lc}
\hline & Frecuencia \\
Estudio de mercado & .79 \\
Red de proveedores y distribuidores & .76 \\
Dist. de clientes por segmento o negocio & .75 \\
Relaciones con clientes & .75 \\
Compromiso con clientes & .72 \\
Captación nuevos clientes & .72 \\
Alianzas estratégicas, acuerdos, socios estratégicos & .68 \\
Inversiones en nuevos mercados & .68 \\
Actividades de marketing & .61 \\
Diversificación de mercados & .56 \\
Adquisiciones / participaciones & .53 \\
Exportación de productos & .49 \\
Estrategia de información y comunicación & .44 \\
Esfuerzos relativos ambiente de trabajo & .39 \\
Producción cliente & .24 \\
Posición de liderazgo internacional & .15 \\
Dependencia del cliente clave & .14 \\
Clientes web & .14 \\
Educación formación de clientes & .11 \\
Clientes / empleado & .11 \\
\hline
\end{tabular}

Fuente: Elaboración propia siguiendo los resultados de sPss

El cuadro 5 detalla los resultados del capital relacional, aspecto que contempla las relaciones de la empresa con clientes, competidores, accionistas, así como otros agentes. El capital relacional ocupa el segundo lugar en la divulgación de los activos intangibles para las empresas mexicanas. Los aspectos más destacados por divulgar se encuentran entre un $70 \%$ y $80 \%$, los cuales corresponden a los siguientes ítems: estudio de mercado, red de proveedores y distribuidores, distribución de clientes por segmento o negocio, relaciones con clientes, compromiso con clientes y captación de nuevos clientes. Los ítems menos valorados se encuentran entre un $10 \%$ y $15 \%$, que corresponden a posición de liderazgo internacional, dependencia del cliente clave, clientes web, educación, formación de clientes y cliente empleado. 


\section{Cuadro 6 \\ Revelación de información sobre capital humano}

\begin{tabular}{lc}
\hline & Frecuencia \\
\hline Experiencia equipo directivo & .92 \\
Políticas incentivos / compensación & .69 \\
Contrato colectivo trabajo & .63 \\
Experiencia empleados & .61 \\
Capacidad adiestramiento a empleados & .60 \\
Pensiones & .57 \\
Posesión acciones empleados directivos & .50 \\
Calidad equipo directivo & .38 \\
Políticas de educación y formación & .35 \\
Sistemas de remuneración & .32 \\
Dependencia personal clave & .32 \\
Cultura corporativa & .23 \\
Políticas de reclutamiento & .22 \\
Oportunidades rotación empleo & .20 \\
Oportunidades hacer carrera & .19 \\
\hline
\end{tabular}

Fuente: Elaboración propia siguiendo los resultados de sPSS

En el capital intelectual, el capital humano es el aspecto más importante porque forma la parte fundamental del conocimiento. Para las empresas mexicanas, el capital humano ocupa el último lugar en la divulgación de los activos intangibles. El cuadro 6 evidencia los aspectos importantes que se encuentran en un rango de entre $60 \%$ a $92 \%$; se destaca primeramente la experiencia del equipo directivo; las políticas, incentivos y compensación; el contrato colectivo del trabajo; la experiencia de los empleados y la capacitación y el adiestramiento de los trabajadores. Los ítems menos importantes se encuentran entre un rango de $19 \%$ a 32\%, los cuales corresponden a sistemas de remuneración, dependencia personal clave, cultura corporativa, políticas de reclutamiento, oportunidades de rotación de empleo y oportunidades de hacer carrera.

Como resultado del análisis descriptivo realizado al capital intelectual, la estrategia general de las empresas mexicanas consiste en advertir a los inversionistas los riesgos que conllevan el invertir en ellas de acuerdo con la capacidad de operación reflejada en sus instalaciones de punta, en la forma en que desarrollan la eficiencia de su gobierno, en la calidad de sus productos, en la relación que tienen en el mercado con sus proveedores y clientes, y en la forma en que desarrollan estos 
escenarios con base en la experiencia de sus directivos y de sus empleados; esto garantizará la confianza de sus inversionistas.

\section{Análisis estadístico}

Posteriormente, y para contrarrestar la información antes obtenida, se realizó este análisis estadístico, que abordó todos aquellos contrastes posibles para los diversos niveles de las variables independientes, lo que nos permitió identificar qué variables son lo suficientemente significativas en la divulgación del capital intelectual.

Por tal motivo, y para determinar qué factores eran los que influían en las empresas mexicanas para divulgar el capital intelectual, se contrastó la variable dependiente con las variables independientes: cifra de negocio ( $\mathrm{LnCNeg}$ ), activo (Act), número de empleados (NE), sector productivo (Sector), cotización en mercado extranjero (CotMExt), variable pérdidas (VarPérdida), rentabilidad financiera (RFin) y endeudamiento (Ende).

Para este análisis se utilizó la técnica inferencial de Kolmogorov-Smirnov; esta prueba nos permitió comprobar si la distribución de las frecuencias de una variable obtenida en una muestra es compatible con una determinada distribución de probabilidad que se fija sobre una base teórica verificándose, de esta manera, la normalidad y homoscedasticidad de las variables. Como resultado de este análisis se aplicaron pruebas no paramétricas debido a la falta de normalidad de las variables.

Una vez determinada la prueba estadística, consideraremos la prueba de MannWhitney, la cual se aplicó a las variables dicotómicas que corresponden a la cotización en el mercado extranjero y a las pérdidas.

\section{Cuadro 7}

Resultados de las variables con la prueba Mann-Whitney

\begin{tabular}{|c|c|c|c|c|}
\hline \multirow{2}{*}{ Variable independiente } & \multicolumn{2}{|c|}{ Rangos promedio } & \multirow{2}{*}{$\begin{array}{l}\text { Sig. Asintót } \\
\text { (bilateral) }\end{array}$} & \multirow[b]{2}{*}{$\mathbf{Z}$} \\
\hline & $\mathbf{0}$ & 1 & & \\
\hline $\begin{array}{l}\text { Cotizan mercado } \\
\text { extranjero }\end{array}$ & 48.31 & 55.86 & .237 & -1.183 \\
\hline Variable pérdidas & 52.56 & 38.83 & .091 & -1.691 \\
\hline
\end{tabular}

Fuente: Elaboración propia siguiendo los resultados de spss 
En el cuadro 7 se encuentran los resultados obtenidos para estas dos variables. El rango medio de las empresas que no cotizan en el mercado extranjero es igual a 48.31, mientras que las que cotizan es superior 55.86; no obstante, las diferencias no son estadísticamente significativas, lo cual se comprueba con el resultado que presenta la prueba de un $p=0.237$; por lo tanto, la estrategia de incursionar en mercados internacionales no es primordial para que las empresas divulguen su información voluntariamente. También se observa que las empresas que muestran pérdidas son menos vulnerables a divulgar los activos intangibles, aunque esta variable no muestra una influencia significativa porque el resultado que indica es de $p=0.091$, lo que comprueba que las empresas mexicanas no utilizan esta táctica para brindar seguridad a sus inversionistas.

Para el resto de las variables se aplicó la prueba de Kruskal-Wallis, cuyo objetivo consistió en comparar la tendencia central de varias poblaciones o niveles de tratamiento asignando y estableciendo los rangos a las puntuaciones de la variable. Dicha prueba reflejó los resultados que se presentan en el cuadro 8.

\section{Cuadro 8}

\section{Resultados de las variables mediante la prueba de Kruskal-Wallis}

\begin{tabular}{lccc}
\hline \multicolumn{1}{c}{ Variable } & Chi-cuadrado & gl & Sig.asintót. \\
\hline Cifra de negocios & 19.478 & 3 & .000 \\
Activo & 11.048 & 3 & .011 \\
Número de empleados & 18.717 & 3 & .000 \\
Rentabilidad financiera & 7.129 & 3 & .068 \\
Endeudamiento & 7.107 & 3 & .069 \\
\hline
\end{tabular}

Fuente: Elaboración propia siguiendo los resultados de sPss

Los resultados univariantes revelaron que las variables proxy del tamaño empresarial: cifra de negocios $\left(\mathrm{X}_{2}(3)=19.478, p=0.000\right)$, activo $\left(\mathrm{X}_{2}(3)=11.048\right.$, $p=0.011)$ y número de empleados $\left(\mathrm{X}_{2}(3)=18.717, p=0.000\right)$ mostraron una asociación estadísticamente significativa con la revelación de datos sobre intangibles, determinando con esto que cuanto mayor es la cifra de negocios, el activo y el número de empleados de las empresas mexicanas mayor será su divulgación de capital intelectual, lo que resulta acorde con la literatura previa y a nuestras expectativas de estudio. Las variables rentabilidad y endeudamiento sólo mostraron un nivel de significatividad del $10 \%$. 
Por otra parte, la variable sector se analiza de manera separada porque con esta prueba se define si la divulgación de capital intelectual presenta niveles de similitud en los seis sectores productivos de las empresas. En el cuadro 9 se muestran los resultados.

\section{Cuadro 9}

Resultados prueba Kruskal-Wallis variable sector

\begin{tabular}{llcc}
\hline & \multicolumn{1}{c}{$\begin{array}{c}\text { Sector } \\
\text { productivo }\end{array}$} & N & Rango promedio \\
\hline Total capital intelectual & Alimentos y bebidas & 18 & 60.69 \\
& Construcción e inmobiliaria & 16 & 60.84 \\
& Industrial & 19 & 59.76 \\
& Comunicación & 17 & 38.53 \\
& Otros & 14 & 36.57 \\
& Textil, calzado y complementos & 16 & 42.59 \\
\hline \multicolumn{1}{c}{ Total } & 100 & \\
\hline
\end{tabular}

Fuente: Elaboración propia siguiendo los resultados de sPSS

El cuadro 9 presenta los rangos medios obtenidos del total de capital intelectual para cada uno de los sectores productivos. Por lo tanto, los sectores observados muestran que existe discrepancia suficiente entre los seis rangos concluyendo que no existe concordancia entre ellos. Los sectores que representan un porcentaje mayor de divulgación son construcción e inmobiliaria, alimentos y bebidas y el industrial, no así los sectores textil, calzado y complementos, comunicación y otros. Asimismo, se revela un resultado de $\mathrm{X}_{2}(5)=13.534, p=0.019$, mostrando de esta manera que existen diferencias estadísticamente significativas entre los sectores analizados de acuerdo con su nivel de divulgación e información sobre capital intelectual.

Los resultados obtenidos de las variables independientes muestran que las variables correspondientes al tamaño de la empresa (cifra de negocio, activo y número de empleados) son estadísticamente significativas, al igual que la variable de sector productivo. Las demás variables no señalan esta situación en las empresas que cotizan en mercados extranjeros y en las empresas que muestran pérdidas, así como las razones correspondientes al financiamiento y endeudamiento, estas dos últimas variables son significativas al $10 \%$. 
Con base en el resultado anterior, y para conocer estadísticamente el factor que más influye en las empresas para que éstas divulguen información, se llevó a cabo el análisis multivariado, utilizando el método estadístico de regresión lineal, el cual estima los coeficientes de la ecuación lineal de cada variable independiente que mejor prediga el valor de la variable dependiente; con este análisis comprobaremos si las variables independientes son capaces de pronosticar o explicar la divulgación del capital intelectual. El cuadro 10 muestra la prueba estadística de regresión lineal. Se incluyó sólo una proxy de tamaño, verificándose la ausencia de multicolinealidad.

\section{Cuadro 10 \\ Regresión lineal capital intelectual \\ Correlación de variables}

\begin{tabular}{lccc}
\hline \multicolumn{4}{c}{ Variable dependiente capital intelectual } \\
\multicolumn{1}{c}{ Variable independientes } & $\mathbf{B}$ & $\mathbf{T}$ & Sig. \\
\hline & $\mathbf{. 4 2 5}$ & $\mathbf{4 . 1 5 2}$ & $\mathbf{. 0 0 0}$ \\
Logaritmo cifra de negocios & -.096 & -.974 & .333 \\
Sector comunicación & .014 & .149 & .882 \\
Cotizan mercado extranjero & $\mathbf{. 2 5 6}$ & $\mathbf{2 . 5 3 7}$ & $\mathbf{. 0 1 3}$ \\
Sector alimentos y bebidas & & & \\
Sector construcción e inmobiliaria & $\mathbf{. 2 8 0}$ & 2.912 & $\mathbf{. 0 0 5}$ \\
Sector industrial & .189 & 1.929 & .057 \\
Endeudamiento & .181 & 1.787 & .077 \\
Rentabilidad financiera & -.153 & -1.477 & .143 \\
Variable pérdidas & -.169 & -1.504 & .136 \\
& & & \\
\hline R Cuadrado & .365 & & \\
R Cuadrado corregida & .302 & & \\
\hline
\end{tabular}

Fuente: Elaboración propia siguiendo los resultados de SPSS

Los resultados multivariantes indican la significatividad de la variable tamaño $(\mathrm{p}<0.01)$ y del sector $(\mathrm{p}<0.05)$, especialmente del sector de alimentos y bebidas y el sector de construcción e inmobiliaria. Los signos positivos de los coeficientes de las variables anteriores revelan que la divulgación de activos intangibles es mayor en las grandes empresas, lo que resulta acorde con los estudios realizados por Cooke (1989), Raffournier (1995) y García-Meca (2005). Las empresas pertenecientes a los sectores de alimentos y bebidas y construcción e inmobiliaria también se muestran más proactivas en la divulgación de estos datos, lo cual podemos 
atribuirlo a su mayor tradición en el mercado mexicano, así como a su experiencia. También es importante destacar la significatividad al 10\% de las variables sector industrial y endeudamiento.

\section{Conclusiones}

Actualmente en México, el organismo encargado de la normatividad contable es el Consejo Mexicano para la Investigación y Desarrollo de Normas de Información Financiera (CINIF), el cual mediante el Boletín "C8" define al activo intangible como: "aquellos activos no circulantes que sin ser materiales o corpóreos son aprovechables en el negocio. Con base en esta definición existen restricciones para reconocer contablemente al activo intangible, pero que de manera voluntaria las empresas pueden reflejarlo. Sin embargo, a la fecha no se tiene una base concreta de cómo son divulgados los activos intangibles en las empresas mexicanas y los factores explicativos de la mayor o menor proactividad de estas empresas en cuanto a la revelación voluntaria de este tipo de datos.

Por lo anterior, a lo largo de esta investigación se han desarrollado los estudios estadísticos que comprueban la divulgación de información del capital intelectual de las empresas mexicanas que cotizan en la Bolsa Mexicana de Valores y sus factores explicativos, de acuerdo con la información obtenida por la propia Bolsa Mexicana de Valores.

Mediante los datos estadísticos descriptivos que fueron utilizados para medir el grado de divulgación del capital intelectual, podemos señalar que los resultados reflejaron que el capital estructural es la categoría sobre la que más se divulga información, seguido por el capital relacional y por último por el capital humano. Lo anterior significa que para las empresas mexicanas es primordial dar a conocer el aspecto interno del conocimiento explicitado, sistematizado e internalizado de la organización, seguido del aspecto de las relaciones con el exterior, ya sea con clientes, proveedores, con el mercado, entre otros, los cuales dependen del personal de la organización. Finalmente, revelan datos sobre el conocimiento, aspecto que el capital intelectual considera de vital importancia por estar relacionado directamente con los recursos humanos.

Las deducciones para las variables que miden el tamaño de la empresa — como es cifra de negocios, activo y número de empleados- reflejan resultados estadísticamente significativos, con lo que confirmamos que cuanto mayor es la cifra de 
negocios, el activo y el número de empleados, mayor son la divulgación de los activos intangibles. Debemos señalar que las empresas de mayor tamaño cuentan con una mayor cobertura de analistas y peso de inversionistas institucionales en su accionariado; como consecuencia, éstas se ven sometidas a una mayor presión para el suministro de información, lo que explicaría que sean más proactivas en la revelación de datos sobre intangibles. Con lo anterior se comprueban las teorías de agencia (Jensen y Mecking, 1976; Myers, 1977) y la teoría de las señales (Ross, 1979), las cuales indican que existe una relación positiva entre el tamaño de la empresa y la extensión de información divulgada

Nuestros resultados univariantes también revelan que las empresas mexicanas más endeudadas y rentables realizan esfuerzos superiores al resto para revelar datos sobre sus activos intangibles. Las empresas más endeudadas se ven sometidas a mayores exigencias informativas por las agencias de rating, lo que justificaría su tendencia a divulgar voluntariamente información sobre intangibles, lo cual también favorece la reducción de su costo de capital al reflejar aquellos generadores de valor no incluidos en su información financiera. Los esfuerzos de los directivos de las compañías más rentables por mejorar la imagen de su empresa, por justificar su rentabilidad y por conducir a una mayor remuneración, también explicarían los resultados obtenidos.

La prueba a la variable del sector productivo se llevó a cabo por separado para conocer los resultados por cada sector, con lo cual podemos corroborar que existen diferencias estadísticamente significativas entre los sectores analizados de acuerdo con su nivel de divulgación e información sobre capital intelectual. Se aprecia que los sectores más proactivos son el sector de alimentos y bebidas y construcción e inmobiliaria, que suelen abarcar empresas con gran tradición y experiencia en México, lo que explicaría la mayor proactividad divulgativa de estos sectores. De igual forma, la teoría de las señales indica que la divulgación de la información de intangibles por la empresa está condicionada por el sector al cual pertenecen.

Los resultados que se obtuvieron evidencian que tanto la variable de cotización en mercado extranjero como la variable de pérdidas no son estadísticamente significativas en la divulgación del capital intelectual. Por tanto, no se aprecian diferencias en la revelación de datos sobre intangibles, según la internacionalización de su capitalización, aun cuando con esto provoque una estrategia de comunicación de mayor calidad y transparencia de imagen en el nivel internacional. 
Del mismo modo, estos resultados serán de utilidad a la comunidad empresarial mexicana porque les permitirá conocer la actuación e importancia del capital intelectual en las mismas, tomando en cuenta los diferentes valores con los que cuentan y de esta manera obtener una mejor posición de valor dentro del mercado. Con esta información, los directivos contemplarán un panorama general, lo que les permitirá la eficiente toma de decisiones que mediante adecuadas estrategias, estudios, proyectos y otros, llevarán a sus empresas a una ventaja competitiva.

Esta primera investigación es el inicio de futuros estudios, que contribuirán a conocer la actuación de las empresas mexicanas con el capital intelectual en años subsecuentes, lo que también permitirá ratificar los resultados obtenidos. Por otra parte, y dada la importancia del tema, es necesario analizar la influencia de las características generadas y de aquella relación con la estructura financiera y el gobierno corporativo, así como estudiar la influencia del capital intelectual en la rentabilidad de las mismas. De esta forma, identificaremos y conoceremos el aspecto de divulgación e influencia del capital intelectual en México.

\section{Referencias}

Abad, C., F. Bravo y M. Trombetta (2007). "Análisis metodológico y empírico de los problemas en la medición de la información voluntaria". Congreso de Asociación Española de Contabilidad y Administración de Empresas (AECA), septiembre.

Adrem, A. (1999). "Essays on disclosure practices in Sweden-Causes and Effects", Lund Studies in Economics and Management 51, The Institute of Economic Research, Sweden: Lund University Press.

Bueno, E., P. Salvador, O. Rodríguez y G.Martín de Castro (2004). "Internal Logic of the Interactions among Intangibles in a Model of Intellectual Capital: The Cognitive Neuron of the Intellectus Model". IC Congress, Helsinki

Bukh, P.N., P. Gormses, y J. Mouritsen (2001). "Intellectual capital statements on their way to the stock exchange", Working Paper, Aarhus School of Business, Aarhus, Denmark. 
CaÑibano, L. (2005)."La auditoría tras la adopción de las Nic/NIIF. Valor razonable, intangibles, transparencia y buen gobierno". Partida Doble, $\mathrm{N}^{\mathrm{o}}, 169$, pp. 28-53.

, A. Gisbert, E. Garcia-Meca y B García-Osma(2008). "Los intangibles en la regulación contable", Documento AECA \& Instituto Análisis Intangibles, Madrid.

, M. Calvo, P. Sánchez, M. García-Ayuso y C.Chaminade (2002). "Directrices para la gestión y difusión de información sobre intangibles, Informe del Capital Intelectual”. Proyecto MÉRITUM

Castilla, P. y D. Gallardo (2007). "Una revisión al estado de divulgación de Intangibles, motivos, contenidos e informes más utilizados”. Comunicación presentada en el congreso de AECA, septiembre.

Cooke, T. (1989). "Disclosure in the corporate annual reports of Swedish Companies", Accounting and Business Research, julio, pp. 533-541.

Devece, C., D. Palacios y F. Garrigón (2007). "Un estudio DelPHi para la medición del capital intelectual en la empresa". Documento de trabajo. Castellón.

Edvisson, L. y M. S. Malone (1997). "El capital intelectual, cómo identificar y calcular el valor de los recursos intangibles de su empresa". GESTION 2000 .

Euroforum (1998). "Medición del capital intelectual: modelo Intelect”. Madrid: I.U. Euroforum Escorial.

Frank, M. y V. Goyal (2003). "Testing the Pecking Order Theory of Capital Structure”, Journal of Financial Economics, 67(2), pp. 217-248.

García Arrieta, M. (2001). "La información contable de los activos intangibles" Tesis Doctoral, Madrid: Universidad San Pablo CEU. 
García-Meca, E. (2003).'El capital intelectual en el mercado de capitales español: La divulgación en canales privados y el reflejo en los informes de analistas financieros". Tesis Doctoral, Universidad de Murcia.

I. PARra, I. Martínez y M. Larrán, (2005). "The explanatory factors of intellectual capital information to financial analysts", European Accounting Review, Vol.14, N. 1, pp.63-95.

e I. Martinez, (2005). "The Relevance on Intellectual Capital Information in Investment Decision. An empiricall study using analyst reports", International Journal of Accounting, 42 (1), pp. 57-81

(2005). "Bridging the gap between disclosure and use of Intellectual capital information". Journal of Intellectual Capital, Vol. 6, Nº, 3, pp.427440 .

Gray, D., T. Rastas y G. Roos (2004). What Intangible resources do Companies Value, Measure and Report? A Combination of UK and Finnish Research". IC Congress, Helsinki.

Harris, M. y A. Raviv (1991). "The Theory of Capital Structure", Journal of Finance, 46, 1, pp. 297-355.

Jensen, M. y W.H. MecKing(1976). "The theory of firm: managerial behavior, agency costs and ownership structure", Journal of Financial Economics, Vol. 3, October, pp. 305-360.

Laínez, J. A., S. Callao y J.I. Jarne (1999). “Tendencias en la presentación de la información económico-financiera en las empresas europeas", X Congreso AECA, septiembre, Zaragoza.

LEv, B. (2000). "New measures for the new economy. Disponible en: http://www. stern.nyu.edu/-blev.

MeeK, G., C.Roberts y S. Gray (1995). "Factors influencing coluntary annual reports disclosures by US and UK and Continental European Multinational Corporations", Journal of International Business Studies, Vol. 26, pp. 555-572. 
MYERS, S.C. (1977). "Determinants of corporate borrowing". Journal of Financial Economics, 5, 147-176.

Monclús, R., A. Rodríguez, T. Torres y M. Vidal (2007) "Prácticas Informativas sobre capital humano. Estudio empírico en las empresas cotizadas en el mercado continuo español". Comunicación presentada al II Congrès Accid 24-25 mayo. http://www.accid.org/congres/historic/2007/ponencies/np007.pdf

NAvas, J. y M. Ortiz (2002). "El capital intelectual en las empresas- análisis de criterios y clasificación multidimensional. Revista Economía Industrial No. 436 pp.. 163-171

RodríGuez, G. (2004). "Factores explicativos de la revisión voluntaria de información: Sobre fuentes de ventaja competitiva empresarial". Revista Española de Financiación y Contabilidad, Vol. XXXIII n ${ }^{\circ}, 122$, julio - septiembre 2004, pp. $705-739$.

Ross, S. A., (1979). "Disclosure regulation in the financial markets: implications of modern finance theory and signaling theory", Issues in Financial Regulation, New York: McGraw-Hill.

Sveiby, K. (1997). "Capital Intelectual: La nueva riqueza de las empresas - cómo medir y gestionar los activos intangibles para crear valor", GESTION 2000

Tecce, D. (2000). "Managing Intellectual Capital: Organizational, Strategic and Policy Dimensions". Managing Intellectual Capital 2002.

Villacorta, M. (2005). "Revelación de la Información voluntaria sobre el capital humano en los informes anuales". Revista Intangible Capital, enero - marzo, año/Vol. 2, no, 011, pp. 37 - 71.

http://portal.imcp.org.mx/content/view/1535/42/ Normas de Información Financiera Instituto Mexicano de Contadores Públicos.

http://www.bmv.com.mx/BMV/JSP/sec5_infogral.jsp Página Bolsa Mexicana de Valores. 


\section{ANEXO \\ Índices revelación de intangibles a evaluar en reportes anuales}

Capital estructural (23)

Calidad de sus productos

Capacidad instalada/ tecnología de punta

Certificados / reconocimientos calidad

Cuota mercado

Diversidad nuevos productos /tecnología

Eficiencia productiva

Estructura accionarial

Estructura control interno

Estructura organizativa

Gestión del modelo de negocio

Gobierno corporativo

Inf. sobre imagen, líder y marcas

Inf. sobre visión del negocio

Políticas, objetivos, estrategias I+D+I

Políticas /normativas medioambientales

Reconocimiento activos intangibles

Responsabilidad social corporativa

Riesgos de la empresa

Servicios sociales

Sistemas e inversiones de IT

Sistemas integrales de información

Utilización de energía y otros inputs

Ventaja competitiva

Capital humano (15)

Calidad equipo directivo

Capacitación adiestramiento empleados

Contrato colectivo de trabajo

Cultura corporativa

Dependencia del personal clave

Experiencia del equipo directivo

Experiencia empleados

Oportunidades de hacer carrera

Oportunidades de rotación de empleo

Pensiones

Política de educación y formación

Política incentivos / compensaciones

Política de reclutamiento

Posesión acciones empleados/directivos

Sistemas de remuneración 


$\begin{array}{ll}\text { Capital Relacional (20) } & \text { Actividades en marketing } \\ \text { Adquisiciones / participaciones } & \text { Alianzas estrat. acuerdos socios estrat. } \\ \text { Capacitación nuevos clientes } & \text { Clientes web } \\ \text { Clientes / empleado } & \text { Compromiso con clientes } \\ \text { Dependencia del cliente clave } \\ \text { Dist. clientes por segmento o negocio } \\ \text { Diversificación de mercados } \\ \text { Educación /formación de clientes } \\ \text { Esfuerzos relativos ambiente trabajo } \\ \text { Estrategia de información y comunicación } \\ \text { Estudios de mercado } \\ \text { Exportación de productos } \\ \text { Inversiones en nuevos mercados } \\ \text { Posición de liderazgo internacional } \\ \text { Producción / cliente } \\ \text { Red de proveedores y distribuidores } \\ \text { Relación con clientes }\end{array}$

Fuente: Elaboración propia G 